\title{
Bases científicas del pensamiento geológico de Benito Jerónimo Feijoo
}

Scientific foundations of the geological thought of Benito Jerónimo Feijoo

Cándido Manuel García Cruz

International Commission on the History of Geological Sciences

CESXVIII, núm. 27 (2017), págs. 197-218 DOI: https://doi.org/10.17811/cesxviii.27.2017.197-218

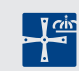




\section{RESUMEN}

Benito Jerónimo Feijoo, intelectual, erudito y ensayista de la Ilustración española durante la primera mitad del siglo XVIII, realizó una gran aportación en el campo de la ciencia geológica de los temas más controvertidos del momento a través de un análisis reflexivo y de su divulgación. Abarcó el origen de la Tierra bajo la autoridad de las Sagradas Escrituras frente a los torbellinos cartesianos; intuyó que el geomagnetismo procedía de la zona central del planeta; consideraba que los fósiles eran de naturaleza orgánica, negando al mismo tiempo la existencia de los gigantes; los terremotos y los volcanes se debían a fenómenos físico-químicos y eléctricos; defendía la existencia de las montañas antes del Diluvio y su lenta formación mediante el espíritu lapidífico; y era partidario de que los puentes intercontinentales habían sido el mecanismo para el poblamiento de América y la dispersión de los seres vivos. A pesar del enfoque racional en sus discusiones, siempre estuvo sujeto al dogma católico, y consideraba compatibles ambos puntos de vista. Sus fuentes de documentación fueron principalmente obras francesas y de otros autores escritas en latín.

Palabras clave

Feijoo, fósiles, montañas, terremotos, volcanes, geomagnetismo, puentes intercontinentales.

\section{Abstract}

Benito Jerónimo Feijoo, an intellectual, polymath, and essayist of the Spanish Enlightenment during the first half of the 18th Century, made a major contribution in the field of geological science of the most controversial issues of his time by means of a thoughtful analysis and its spreading. He covered the origin of the Earth under the authority of Holy Scripture against the Cartesian vortices; was convinced that the geomagnetism came from the central zone of the planet; believed that fossils were organic in nature, denying at the same time the existence of the giant humans; earthquakes and volcanoes were due to physical-chemical and electrical phenomena; supported the existence of the mountains before the Flood and its slow formation as a result of the lapidific spirit; and was an advocate of the land bridges as a mechanism for both the human settlement of America and the dispersion of living beings. Despite the rational approach in his discussions, he was always subject to Catholic dogma, considering both as compatible points of view at all. Feijoo's sources of documentation were mainly French authors and other sources written in Latin.

KeY WoRDS

Feijoo, fossils, mountains, earthquakes, volcanoes, geomagnetism, land bridges.

Recibido: 20 de marzo de 2017. Aceptado: 21 de abril de 2017. 


\section{Introducción}

El monje benedictino gallego Benito Jerónimo Feijoo (1676-1764), catedrático de Teología de la Universidad de Oviedo, fue, sin duda, uno de los intelectuales más relevantes de la Ilustración española, ensayista y erudito en muchos campos del saber, tanto de las humanidades como de las ciencias $^{1}$. Su obra fundamental, y además controvertida, el Teatro crítico universal, en ocho volúmenes publicada a lo largo de trece años (1726-1739), con casi 3.500 páginas, se compone de 121 discursos o ensayos escritos, según se lee en la página de título de este tratado, «para desengaño de errores comunes», sobre las más variadas materias, desde el derecho, la historia, la política y la economía, hasta la física y la química, las ciencias naturales y la astronomía, pasando por la filosofía, las matemáticas, la literatura o la geografía, así como la filología, la religión o la medicina. A este libro siguieron, entre 1742 y 1760, las Cartas eruditas y curiosas, en cinco volúmenes, con 162 cartas en algo más de 2000 páginas, donde se continuaba lo proyectado en el Teatro, «impugnando, o reduciendo a dudosas, varias opiniones comunes». A pesar de estas intenciones feijonianas, tal y como ya ha sido señalado y enfatizado por diversos estudiosos, España no era en esa época «un desierto sumido en las más profundas tinieblas» ${ }^{2}$, ni se encontraba «en el misérrimo estado de ignorancia, barbarie y fanatismo» ${ }^{3}$ al que pretendía atacar enérgicamente el monje gallego: el Movimiento Novator en la España de finales del siglo XVII y principios del XVIII ya había efectuado

1 Sobre la vida de Feijoo, remitimos a los aspectos biográficos que fueron recogidos en 1765 por Pedro Rodríguez de Campomanes (1723-1802), y publicados en una nueva reimpresión del primer volumen del Teatro crítico universal, aunque no figura como su autor; véase Pedro Rodríguez Campomanes, «Noticia de la vida, y obras del M. I. y R. P. D. Fr. Benito Jerónimo Feijoo», en B.J. Fejoo, Teatro crítico universal, Madrid, Real Compañía de Impresores y Libreros, 1778, t. I (nueva impresión), págs. I-XLVI; sobre su autoría, véase Inmaculada Urzainqui, «Campomanes y su "Noticia” de Feijoo", en R. Morales Raya (coord.), Homenaje a la profesora M. ${ }^{a}$ Dolores Tortosa Linde, Granada, Servicio de Publicaciones de la Universidad de Granada, 2002, págs. 481-492.

2 José Luis Abellán, Historia crítica del pensamiento español, t. 3: Del Barroco a la Ilustración, siglos XVII-XVIII, Madrid, Espasa-Calpe, 1981, pág. 491.

3 Marcelino Menéndez Pelayo, Historia de los heterodoxos españoles, Madrid, CSIC, 1882 (facsímil 1992), vol. II, Libro VI, cap. 1, vI, pág. 518. 
aportaciones importantes en el desarrollo y en la difusión cultural en las décadas precedentes ${ }^{4}$.

En realidad, Feijoo es heredero y tributario de estos Novatores, y como tal procede en sus escritos, discutiendo y analizando gran número de aspectos culturales que le preocupan, y con un espíritu crítico que aplicó no solo a la historia, sino a todas las parcelas del conocimiento, con el rigor y la profundidad que le permitía su docto saber. No poseía estudios formales o académicos en ninguna rama de la ciencia; sin embargo, como un auténtico polígrafo, actuó con una erudición poco habitual en relación con las ciencias naturales, incluyendo aquí también la física y la química ${ }^{5}$. No se le puede considerar un científico al uso en tanto que no realizó ningún descubrimiento que tuviera que ver con cualquier campo de la ciencia. Pero como lector infatigable, su curiosidad, sus ansias de conocimiento, y su posición frente a la filosofía aristotélica, le llevaron a hacer una gran contribución al convertirle en el gran impulsor del estudio de la naturaleza, uno los principales promotores de la creación de sociedades e instituciones estatales, y al mismo tiempo de una reforma imprescindible de la universidad española ${ }^{6}$, el mejor maestro para la divulgación y la difusión de las ciencias naturales a través de sus reflexiones, con un juicio crítico basado en el racionalismo y en la experiencia (TC, VII, 13, § XI, 35), considerada esta última como «la regla matemática de la fe humana» y una gran maestra (TC, V, 1 y TC, V, 11), frente a las supersticiones y las creencias erróneas de las ideas escolásticas. No obstante, siempre estuvo sometido al dogma católico como referencia última, a lo que él consideraba la infalible verdad de la Historia Sagrada (TC, V, 15, § VII, 15). Esto no le impidió acercarse a los conocimientos de una época en

4 A modo de introducción sobre los novatores en España, véanse, por ejemplo, AbELLÁN, Historia crítica, págs. 342-461; Julián LóPEZ CRUCHET, «Los novatores españoles del siglo XVIII y la revisión del pensamiento escolástico», en Xavier Agenjo Bullón, Rafael V. Orden Jiménez y Antonio Jiménez García (coords.), Nuevos estudios sobre historia del pensamiento español. (Actas de las V Jornadas de Hispanismo Filosófico, Santander, 16-18 abril de 2001), Madrid, Fundación Larramendi, 2005, págs. 109-120; Jesús Pérez MAGALLón, Construyendo la modernidad: la cultura española en el tiempo de los novatores (1675-1725), Madrid, CSIC, 2002.

Algunos estudios sobre la ciencia en Feijoo, con enfoques distintos, fueron llevados a cabo por Miguel Morayta y Sagrario, El padre Feijóo y sus obras, Valencia, F. Sempere Ed., 1912, cap. Ix; y Narciso PÉrEz, «El padre Feijóo y las Ciencias Naturales. Un capítulo de Historia de la Ciencia española», Revista de la Real Academia de Ciencias Exactas, Físicas y Naturales, vol. 41, 1 (1947), págs. 119-173; vol. 41, 2 (1947), págs. 287-337; vol. 41, 3 (1947), págs. 469-513; y vol. 41, 3 (1947), págs. 599-644. El trabajo de Morayta y Sagrario ha sido considerado (creemos que erróneamente) algo superficial por PÉREz, «El P. Feijoo y las Ciencias», pág. 120.

6 Sus críticas al sistema universitario español se encuentran en diferentes partes de su Teatro crítico universal (TC, VII, 11-14, y TC, VIII, 3) y en sus Cartas eruditas y curiosas (CE, II, 16, y CE, III, 31); véase, además, Antonio Álvarez de Morales, La «Ilustración» y la reforma de la universidad en la España del siglo XVIII, Madrid, Instituto de Estudios Administrativos, 1971, cap. 2, págs. 43-55 (preferentemente págs. 44-46). 
la que destacaban las controversias y debates sobre multitud de temas prácticamente en todos los campos de la ciencia, donde es posible subrayar algunos hechos relevantes: la química ya había iniciado el camino hacia la modernización y su independencia de la alquimia que terminaría con el abandono de la teoría del flogisto; la física newtoniana y la revolución astronómica se consolidaban haciendo estragos en el pensamiento filosófico y teológico; la biología, dentro del ordenamiento linneano, discutía, entre otros temas, los presupuestos básicos de las ideas organísmicas frente a las mecanicistas, mientras que la geología intentaba liberarse de la Teología Natural mediante una perspectiva basada en la interpretación racional, observacional y empírica del Libro de la Naturaleza, poniendo por lo tanto más énfasis en las leyes naturales al tiempo que se discutían las enseñanzas escriturales y la tradición hexameral.

De los diversos temas que Feijoo trató en las obras citadas, lo que nos interesa en este trabajo son sus reflexiones críticas sobre el conocimiento geológico. A través de las ideas científicas que considera en sus escritos, y en las que se fundamentan sus discusiones, se pretende analizar cuáles fueron sus fuentes de documentación y sus autores, cosa que, en buena parte y siguiendo la costumbre de la época, no se hacían, por lo general, del todo explícitos. A este respecto, es difícil aceptar la opinión de algunos estudiosos sobre las fuentes que manejó el monje benedictino, al menos en el campo de la ciencia, en el sentido de que tan solo había utilizado misceláneas, revistas y enciclopedias ${ }^{7}$ francesas que le mantenían al corriente de los aspectos culturales de su tiempo ${ }^{8}$. Como veremos, Feijoo hizo uso, además, de diversos trabajos que, si bien es cierto que en su mayoría eran franceses, correspondían a autores notables y de gran predicamento en su tiempo.

\section{Feijoo y el pensamiento geológico de la Ilustración}

En el pensamiento geológico durante el siglo XVII se observa una dependencia o continuidad de las ideas clásicas y medievales a pesar de que conceptos como teoría y práctica van a sufrir grandes cambios ${ }^{9}$, y que pasarán como herencia cultural a la Ilustración. Consecuentemente, este pensamiento relativo

\footnotetext{
7 Las referencias que cita Feijoo en este sentido son Mémoires de l'Académie Royal de Sciences de Paris, Journal de Sçavans, y Mémoires o Journal de Trévoux (cuyo verdadero título era Mémoires pour l'Histoire des Sciences \& des beaux Arts), así como el Dictionnaire Critique et Historique de Pierre Bayle.

8 Abellán, Historia crítica, pág. 498. De las críticas contemporáneas que recibió al respecto, el monje gallego hizo su propia defensa (TC, III, «Prólogo apologético»).

9 Roger ARIEw, «A new science of geology in the Seventeenth Century?», en Peter Barker y Roger Ariew (eds.), Revolution and continuity: Essays in the history and philosophy of early modern science, Washington D. C., Catholic University of America Press, 1991, págs. 81-93.
} 
a la Tierra durante este período va a recoger como patrimonio de la centuria precedente el reconocimiento, la identificación, la explicación y la valoración de un conjunto de objetos materiales (estratos, rocas, minerales, fósiles...) y de conceptos (principios, leyes, teorías...), que servirían para modelar con el paso del tiempo la geología, hasta llegar a consolidarse durante el siglo XIX como ciencia independiente ${ }^{10}$. También se heredarían, por otro lado, grandes controversias como por ejemplo el debate sobre el origen de la Tierra, el geomagnetismo como fenómeno general, el Diluvio bíblico en tanto que agente geomorfológico y como acontecimiento de entidad universal, con el Catastrofismo y el Neptunismo como corrientes de pensamiento predominantes, en relación directa a su vez con la discusión sobre el origen de las montañas, la antigüedad del mundo, y el significado de los fósiles. Todas estas ideas se plasmarían en trabajos que recibieron en su mayoría la denominación genérica de teorías de la tierra, y que serían la semilla para la concreción de la idea de naturaleza y la asunción de las sensibilidades históricas, especialmente por parte de aquellas teorías que no estaban sometidas a la Física Sagrada y que se fundamentaban en las causas naturales y en el abismo del tiempo ${ }^{11}$. A esto hay que añadir las explicaciones sobre el poblamiento del Nuevo Mundo mediante la teoría de las conexiones terrestres o puentes intercontinentales, que habrían sido interrumpidos por catástrofes, como la irrupción de una gran inundación que habría dado lugar, por ejemplo, a la apertura de la cuenca del océano Atlántico. También se recibieron, con la ayuda de los químicos, las teorías que explicaban las causas de los terremotos, siempre en relación con el origen de los fuegos subterráneos y las erupciones volcánicas, basadas una vez más en fenómenos naturales y, por ende, ajenos a la intervención de la Providencia Divina.

$\mathrm{Y}$ no es posible obviar, por otro lado, que todos estos conocimientos geológicos estaban inscritos en una cronología bíblica según la cual el mundo fue creado el año 4004 a.C., publicada por James Ussher (1581-1656) en sus Annales Veteris Testamenti, en 1650, marco cronológico que, a pesar de que su autor era anglicano, había tenido un cierto eco también en otros círculos católicos, por lo que la Tierra tendría, en la época de Feijoo, algo menos de seis mil años de antigüedad.

Todas estas discusiones, con mayor o menor profundidad, las abarcó Feijoo en sus escritos. En los apartados que siguen se analizarán sus ideas y sus fuentes documentales.

10 Evaristo Álvarez Muñoz, Filosofía de las ciencias de la tierra. El cierre categorial de la geología, Oviedo, Pentalfa, 2004, parte I.

11 Cándido Manuel García Cruz, «Aproximación a las sensibilidades históricas y teorías de la Tierra: De la fe a la razón», Llull, vol. 37, 80 (2014), págs. 87-111. 
La aparición en 1644 de los Principia philosophice de René Descartes (15961650) condicionó el pensamiento filosófico a partir de la segunda mitad del siglo XVII. Esta obra supuso la primera aproximación a la reconstrucción del origen de la Tierra y sobre su evolución desde una perspectiva mecanicista mediante torbellinos o vórtices. Tales ideas se remontaban en realidad a 1633, cuando el filósofo francés termina de escribir Le monde ou Traité de la lumière ${ }^{12}$, libro que no aparecería en vida de su autor, dado que a mediados de ese año se había producido la condena de Galileo por parte de la Iglesia de Roma, por lo que Descartes, prudentemente, decidió retirarla de la imprenta; no se publicaría hasta 1664. No obstante, algunas de estas ideas cartesianas aparecieron resumidas en su Discours de la méthode ${ }^{13}$, en una primera edición, por otro lado, anónima, de 1637.

La idea fundamental de Descartes en relación con el origen del Sistema Solar es que el universo está formado por materia de distintos elementos y de naturaleza líquida que gira formando remolinos circulares, vórtices o torbellinos ${ }^{14}$. Una parte importante de esta materia dio lugar al Sol, con una localización central, mientras que otros torbellinos de menores dimensiones, arrastrados por el propio Sol, originaron los planetas que a su vez formaron los satélites ${ }^{15}$, cuyos movimientos también estaban condicionados por dichos vórtices. Por otro lado, en cuanto a la Tierra, en un principio era un astro formado por materia del primer elemento totalmente pura, como el Sol, pero su aire se fue espesando y dando lugar a cuerpos oscuros que la cubrieron y ocultaron, disminuyendo la fuerza de su torbellino. Esto originó un planeta en enfriamiento progresivo, con regiones bien diferenciadas, que colapsó lentamente, y en el que se mezclaron los distintos elementos que la componían hasta adquirir una estructura interna dividida en distintas zonas, con las irregularidades orográficas en la superficie como resultado de dicha contracción ${ }^{16}$.

12 René Descartes, El mundo. Tratado de la luz, Barcelona / Madrid, Anthropos / Mec, 1989 (facsímil de 1664), [caps. vi-x]; René Descartes, Principes de la Philosophie, en Charles Adams y Paul Tannery (eds.), Oeuvres de Descartes, París, J. Vrin, 1989 (1604), III, 33-63 (trad. francesa de 1904) (en castellano, Guillermo QuinTÁs (trad.), Los principios de la filosofía, Madrid, Alianza, 1995).

13 René Descartes, Discurso del método, Madrid, Alfaguara, 1987 (original de 1637), págs. 30-43.

14 Descartes, Principes de la philosophie, III-IV; sobre la teoría de los torbellinos cartesianos, véanse Eric J. Aтton, «The Cartesian vortex Theory», en René Taton y Curtis Wilson, (eds.), The general history of astronomy, vol. 2: Planetary astronomy from the Renaissance to the rise of astrophysics, Cambridge, Cambridge University Press, 1989, vol. 2, págs. 207-221; Stephen Gaukroger, Descartes' System of Natural Philosophy, Cambridge, Cambridge University Press, 2002, cap. 5, págs. 150-153; Noriss S. Hetherington, Planetary motions: A historical perspective, Westport (CT), Greenwood, 2006, caps. 17-18.

15 Descartes, Principes de la philosophie, III, 33.

16 Descartes, Principes de la philosophie, IV. 
A pesar de que no menciona explícitamente ninguno de los tratados de Descartes citados con anterioridad, sin duda Feijoo los conocía perfectamente, como se deriva de su refutación sobre la base teológica que le proporciona la autoridad de las Sagradas Escrituras, pero también desde un punto de vista racional ${ }^{17}$, el origen y la formación de la Tierra según esta teoría cartesiana de los torbellinos, así como otras consideraciones sobre la obra del Creador (TC, I, 13). En contra de la visión de Descartes en relación con el origen del mundo, el benedictino sostiene que «Dios formó desde el principio la tierra, y los Planetas en el mundo que hoy se ven, sin fiar tales obras al ciego movimiento de la materia» (TC, I, 13, § III, 12). Para Feijoo, incluso, la teoría atomista corpuscular de Pierre Gassendi (1592-1655) sería más aceptable que las propuestas cartesianas, aunque también la consideraba inconsistente (TC, $\mathrm{I}, 13, \S \mathrm{IX})^{18}$.

\section{Sobre el magnetismo terrestre}

La primera descripción de la Tierra como un gran imán se encuentra en la obra De magnete (1600), de William Gilbert (1544-1603), el primer científico experimental o el último gran mago natural, según el punto de vista con el que se analicen sus trabajos.

17 Esta compatibilidad entre la fidelidad al relato bíblico y su discusión racional, ya ha sido señalada por Horacio CAPEL, La Física Sagrada, Barcelona, Ed. del Serbal, 1985, cap. 5, tal como emana de las obras del filólogo y humanista español José Antonio González de Salas (1588-1651), y que trataban sobre las tierras emergidas tras el diluvio; véanse José Antonio González dE SALAS, Dissertacion de la Tierra descvbierta, i cvbierta de las agvas, en Pomponio Mela (c. 40 d. C.), Compendio geographico, Madrid, Diego Díaz de la Carrera Impresor, 1644, págs. [17-42] (en la 2. a impresión: Madrid, Sancha, 1780, págs. 14-61); José Antonio González de Salas, De duplici viventium terra dissertatio paradoxica, Lugduni Batavorum [Leiden], Elzeviros, 1650.

18 Aunque Feijoo tampoco las cita expresamente, las ideas de Gassendi sobre la estructura de la materia se encuentran en la parte Physica de su obra póstuma Syntagma philosophicum, donde el epicureísmo, entre otros aspectos, sustituye al aristotelismo como recurso para explicar el mundo natural; véase Pierre Gassendi, Syntagma philosophicum, en Opera omnia, Lugduni [Lyon], L. Anisson \& I. B. Devenet, 1658, tt. I-II (existe trad. inglesa en: Craig B. Brush (ed.), The selected works of Pierre Gassendi. Nueva York, Johnson Reprint Corp, 1972, págs. 284-434). Véanse, además, Barry Brundell, Pierre Gassendi. From Aristotelianism to a new natural philosophy, Dordrecht, D. Reidel, 1987; Saul Fisher, Pierre Gassendi's philosophy and science. Atomism for empiricists, Leiden / Boston, Brill, 2005, 3. ${ }^{\mathrm{a}} \mathrm{y} 4$. $^{\mathrm{a}}$ partes; Antonia Lolordo, Pierre Gassendi and the birth of early modern philosophy, Cambridge (MA), Cambridge University Press, 2006, preferentemente caps. 5-7; Sylvia Murr, «Pierre Gassendi. Préliminaire à la Physique. Syntagma Philosophicum», XVII Siècle, vol. 179 (1993), págs. 353-385; Margaret J. OsLER, «Becoming an outsider: Gassendi in the history of philosophy», en G. A. J. Rogers, Tom Sorell y Jill Kraye (eds.), Insiders and outsiders in Seventeenth-century philosophy, Nueva York, Routledge, 2010, págs. 23-42. 
Gilbert pensaba, de acuerdo con las ideas herméticas del alma universal, que existía un cierto animismo en todos los componentes del universo ${ }^{19}$. En el caso del globo terráqueo, la fuerza que regía el magnetismo estaba animada o imitaba a un alma ${ }^{20}$.

El benedictino gallego también hace una breve incursión en estos aspectos geofísicos en sus consideraciones sobre el magnetismo terrestre bajo una perspectiva muy distinta, alejándose del animismo hermético. Feijoo (TC, II, 14, § X, 46-49) plantea que la Tierra posee una virtud magnética sobre la base de los experimentos y observaciones realizados con la aguja magnética por Claude François Millet Dechales (1621-1678) ${ }^{21}$. Como explicación racional y mecanicista, sugiere dos mecanismos que en realidad no son excluyentes: de una parte, la corteza terrestre sería una gran cantera de piedra imán, o, de otra, esta virtud magnética se distribuía por todo el globo, con distintos valores de acuerdo con el comportamiento de la aguja en diferentes lugares del planeta. Algunos años más tarde, Feijoo consideraría que la gran piedra imán, con algunos centenares de leguas de diámetro y responsable del magnetismo terrestre, se encontraba cerca del centro de la Tierra ${ }^{22}$.

\section{Sobre los fósiles}

Durante mucho tiempo, el término fósil se utilizó para designar a todo material excavado de la tierra (del latín, fossilis, y éste de fodere, 'excavar', equivalente al griego o@vxtós, 'excavado'), y que abarcaba tanto rocas y minerales como restos orgánicos petrificados. El origen y significado de los fósiles, con

19 William GilBert, De magnete, magneticisque corporibus, et de magno magnete tellure; physiologia nova, plurimis \& argumentis, \& experimentis demonstrata, Londres, Excudebat Petrus Short, 1600, libro IV, cap. 12, págs. 209-210 (existe traducción inglesa de 1893 a cargo de Paul Fleury Mottelay, con edición facsímil de Nueva York, Dover, 1991); véase, además, Stephen Pumfrey, «William Gilbert», en Peter Harman y Simon Mitton (eds.), Cambridge scientific minds, Cambridge, Cambridge University Press, 2002, págs. 6-20.

${ }_{20}$ Gilbert, De magnete, pág. 12. El animismo de Gilbert, y su idea de cuerpo organizado nada tienen que ver, por otro lado, con el organicismo propio de esa época, sino con su creencia en la capacidad de conservación y reparación del planeta de las partes alteradas o dañadas; véase Cándido M. GARCía Cruz, «De la "Teoría de la Tierra" de James Hutton a la "Hipótesis Gaia" de James Lovelock», Asclepio. Revista de Historia de la Medicina y de la Ciencia, vol. 59, 1 (2007), págs. 65-100 (concretamente págs. 76-77).

21 Claude F. M. Dechales, «De magnete», en Cursus seu mundus mathematicus, Lugduni [Lyon], Ex Officina Anissoniana, 1674, t. I, págs. 636-696. Este autor también trató la relación del magnetismo con el sistema copernicano, que a su vez recibiría el análisis crítico de Feijoo (CE, III, 20, 18-20).

22 Texto publicado de forma exenta en Benito J. Feijoo, Nuevo systhema sobre la causa physica de los terremotos, Puerto de Santa María, 1756, pág. 15 (compilado en CE, V, 28, 2). Esta es una idea de una gran modernidad, puesto que la geofísica actual emplaza en la dinámica del núcleo terrestre el origen de dicho magnetismo. 
el sentido de piedras figuradas (lapides figurati) o piedras de aspecto propio (lapides sui generis), ha constituido una de las grandes controversias geológicas durante siglos ${ }^{23}$, si bien en determinados ámbitos, como en la cultura griega o en la árabe, muchos autores pensaban que estas piedras tenían un origen orgánico.

Feijoo define las piedras figuradas como aquellas piedras «que tienen figura propia de algún otro cuerpo de determinada organización específica, como de algún insecto, algún pez, algún ave, alguna planta, algún fruto, algún miembro del cuerpo humano, u otro viviente» (TC, VII, 2, § I, 1). Para explicar su origen, Feijoo descarta la teoría aristotélica de los cuatro elementos, las influencias celestes, y la fuerza petrificante ${ }^{24}$ (Gorgona, virtus fortis mineralium o vis lapidificativa); también desecha que fueran puros juegos de la naturaleza o simples

23 Sobre este tema, siguen siendo de referencia obligada algunos estudios ya clásicos: Frank D. Adams, The birth and development of the geological science, Nueva York, Dover, 1954, cap. v; John G. BurKe, Origins of the science of crystals, Berkeley / Los Ángeles, University of California Press, 1966, cap. II; Francis C. HABER, The age of the world: Moses to Darwin, Baltimore, Johns Hopkins University Press, 1959, caps. II y III; Robert Halleux, Le problème des métaux dans la science antique, París, Belles Letres, 1974, págs. 65-170; Martin J. G. Rudwick, The meaning of fossils. Episodes in the History of Palaeontology, Londres / Nueva York, MacDonald / Elsevier, 1972 (existe edición castellana: Antonio Resines (trad.) El significado de los fósiles. Episodios de la Historia de la Paleontología, Madrid, H. Blume, 1987); Karl A. von ZitTeL, Geschichte der Geologie und Paläontologie bis Ende des 19. Jahrhunderts, Múnich / Leipzig, R. Oldenbourg, 1899, págs. 15-28 (en inglés: Maria M. OGILviE-Gordon (trad.), History of geology and palaeontology to the end of the nineteenth century. Londres / Nueva York, W. Scott / C. Scribner, 1901, págs. 13-23).

Estas ideas a partir del Renacimiento y durante la Ilustración han sido estudiadas por Patricia CREPINOBert, Construction de problèmes et obstacles épistémologiques à propos du concept de fossile: étude épistémologique comparative entre des situations de débat à l'école primaire et au collège et des controverses historiques du XVII au XIXe siècle, Nantes, Cren, 2010; François Ellenberger, Historia de la geología, vol. 1: De la antigüedad al siglo XVII, Barcelona, Labor / MEc, 1989, cap. 3; Jean GaudanT, «John Woodward (1695) et Johann Jakob Scheuchzer (1708): l'irruption du Déluge dans l'interprétation des fossiles», Travaux du Comité Français d'Histoire de la Géologie, 3. ${ }^{\mathrm{a}}$ serie, vol. 22 (2008), págs. 55-63; Jean GAUDANT y Genevieve BoulLLET, «La genèse et l'interprétation de "fossiles" dans la science classique: de la Renaissance aux Lumières", Bulletin de la Société Géologique de France, vol. 171, 5 (2000), págs. 587-601; Yvette GaYrard-Valy, Los fósiles, huellas de mundos desaparecidos, Madrid, Aguilar 1989, cap. 2; Stephen J. Gould, «Father Athanasius on the isthmus of a middle state. Understanding Kircher's paleontology", Athanasius Kircher. The last man who knew everything, Nueva York / Londres, Routledge, 2004, págs. 207-237; Jennifer M. Jordan, “"Ancient episteme" and the nature of fossils: a correction of a modern scholarly error", History and Philosophy of the Life Sciences, vol. 38, 1 (2016), págs. 90-116; Cristina MEschiari, «Gorfon e vis lapidificativa. Elementi di mineralogia tra Alberto Magno e Dante», Saggi e Studi, vol. II, 1 (2014), págs. 1-19; Rhoda RaPPAPORT, When geologists were historians, 1665-1750, Ithaca (NY), Cornell University Press, 1997, págs. 105-135; Paolo Rossi, I segni del tempo: Storia della terra e storia delle nazioni da Hooke a Vico, Milán, G. Feltrinelli, 2003, cap. 1, sec. 1-4 (en la edición inglesa: Lidia G. Cochrane (ed.) The dark abyss of time. The history of the Earth and the history of nations from Hooke to Vico. Chicago, University of Chicago Press, 1984, caps. 1-4); Wim Sissingh, Rocky roads from Firenze. History of geological time and change 1650-1900, Utrecht, Utrecht University, 2012, cap. 3.

24 La idea de la fuerza petrificante, aunque de origen clásico, fue adoptaba durante la Edad Media debido a la influencia de Alberto Magno que la recogió y la divulgó; véanse, por ejemplo, Meschiari, «Gorfon e vis lapidificativa»; Nuno Pimentel, «Visões filosóficas da natureza. A geologia no contexto cultural da Idade Média», Geonovas, vol. 27 (2014), págs. 95-100. Feijoo no cita a Alberto Magno en el contexto de la geología. 
producciones del acaso, así como la teoría de la panspermia y de las semillas petrificantes ${ }^{25}$, ideas todas de tradición muy antigua que hunde sus raíces en las teorías de la materia del mundo clásico ${ }^{26}$. Al mismo tiempo, se decanta por un origen también natural (TC, V, 15, § XVI, 47), pero más acorde con otra de las ideas que predominaron en esa época, a saber, la teoría de los jugos lapidificos (succus lapidiscens), que había sido propuesta por Georgius Agricola (1449-1555) en su tratado De ortu \& causis subterraneorum, a mediados del siglo $\mathrm{XVI}^{27}$. Para analizar estas ideas, Feijoo recurre en especial a fuentes francesas, aunque también a obras escritas en latín, de autores tan renombrados en esa época como Jan Baptist van Helmont (1579-1644), Wilhelm [Guillaume] Homberg (1652-1715), Antoine de Jussieu (1686-1758), Athanasius Kircher (1602-1680), Edward Lhwyd (1660-1709), Etienne-Augustin Souciet (1685I744), y Joseph Pitton de Tournefort (1656-1708).

En los trabajos de estos naturalistas ${ }^{28}$ se planteaba el crecimiento de las

${ }_{25}$ Feijoo (TC, VII, 2, § VIII, 31-32) rechaza incluso la idea defendida por Phillipe de la Hire (16401718) de que los vapores subterráneos ascendían hasta la superficie de las montañas más altas, donde se producía la petrificación; véase Phillipe de la Hire, «Remarques sur l'eau de pluie, et sur l'origine des fontaines; avec quelques particularités sur la construction des citernes», Mémoires de l'Académie Royal de Sciences de Paris, [s. n.] (1703), págs. 56-69.

${ }_{26}$ Véanse, al respecto, Hiro Hiraï, «Le concept de semence de Pierre Gassendi entre les théories de la matière et les sciences de la vie au XvII ${ }^{e}$ siècle», Medicina nei Secoli, vol. 15, 2 (2003), págs. 205-226; Hiro HiRAï, Le concept de semence dans la théorie de la matière à la Renaisance: De Marsile Ficin à Pierre Gassendi, Turnhout, Brepols, 2005; Ingrid D. Rowland, «Athanasius Kircher, Giordano Bruno, and the Panspermia of the infinite universe», en Paula Findlen (ed.), Athanasius Kircher. The last man who knew everything, Nueva York / Londres, Routledge, 2004, págs. 191-205.

27 Georgius Agricola, De ortu \& causis subterraneorum. Libro V: De natura eorum quae effluunt ex terra. Libro IV: De natura fossilium. Libro X: De veteribus \& novis metallis. Libro II: Bermannus, sive De re metallica dialogus. Libro I: Interpretatio Germanica vocum rei metallicae, Basilea, H. Frobenius y N. Episcopius, 1546, págs. 51-57 (existe trad. italiana completa en Venecia, M. Tramezzino, 1550, e inglesa de dos de estos tratados: De re metallica (Bermannus), Nueva York, Dover, 1986, y De natura fossilium (Text of mineralogy), Mineola, Dover, 2004; existen asimismo dos trad. francesas de De re metallica en Thionville, G. Klopp, 1987 y París, Belles Lettres, 1990, y una castellana en Madrid, Círculo Científico, 2004).

28 Jan B. van Helmont, «Tractatus de Lithiasi», en Opuscula medica inaudita, Amsterodami [Amsterdam], L. Elzevirium, 1644, t. I, págs. 9-110; Wilhelm HoмвеRG, «Réflexions sur différentes végétations métalliques», Mémoires de l'Académie Royale des Sciences de Paris, 1692, vol. X, págs. 171-179; Antoine de Jussieu, «Examen des causes des impressions des plantes marquées sur certaines pierres des environs de Saint-Chaumont dans le Lyonnais», Mémoires de l'Académie Royale des Sciences de Paris, [s.n.] (1718), págs. 287-297; Athanasius KIRCHER, Mundus subterraneus, Amsterodami [Amsterdam], Janssonius, 1665, t. II, libro XII, sec. I; Edward LHwyd, Lithophylacii Britannici Ichnographia, Londres / Leipzig, Ex Officina / Gleditsch \& Weidmann, 1699 (versión inglesa en: John Ray, Three physico-theological discourses, Londres, William Innys, 1713, págs. 175-203, con edición facsímil en Nueva York, Arno, 1978; y en Robert Theodore GunTHER, (ed.), Early science in Oxford. Vol. 14: Life and letters of Edward Lhuyd, Oxford, J. Johnson, 1945, carta 200, págs. 381-398); Etienne-Augustin SoucIET, «Dissertation sur les coquillages que l'on trouve dans la Terre», Mémoires pour l'Histoire des Sciences \& des beaux Arts [Mémoires de Trévoux], [s. n.] (février, 1729), págs. 308334; [s. n.] (mars, 1729), págs. 459-484; Etienne-Augustin Souciet, «Seconde Dissertation sur les coquillages que l'on trouve en terre, Mémoires pour l'Histoire des Sciences \& des beaux Arts [Mémoires de Trévoux], [s. n.] 
piedras figuradas mediante la absorción de los mencionados jugos lapidíficos o jugos petrificantes, un concepto, sin duda, mucho más avanzado desde el punto de vista epistemológico que los anteriores de las semillas, la vis formativa o las influencias celestes, y que abarcaba todo tipo de fósiles, desde las piedras figuradas propiamente dichas, hasta las piedras preciosas o gemas, y aquellas otras más comunes.

En relación también con las piedras figuradas, pero atendiendo a otros supuestos orígenes, Feijoo menciona por primera vez, en 1736, unas estructuras cristalinas que se han encontrado en diferentes localidades del Principado de Asturias, como en Las Caldas de Priorio: «Hállanse también en varios parajes piedras de otras figuras. En un sitio distante de esta ciudad una legua, donde llaman las Torres del Priorio, mezcladas con la tierra se encuentran innumerables piedrecillas de tersísima superficie, todas formadas en punta de diamante. En muchas partes se ven cristales hexágonos, estrellados...» (TC, VII, 2, § XVI, 74). En realidad, se trata de una variedad de cuarzo (cuarzo ahumado) que se ha formado a partir de depósitos silíceos en aguas termales ${ }^{29}$. Cuando Feijoo se pregunta a qué principio habría que atribuir estas figuras, descarta muchas ideas que se han visto previamente sobre los fósiles; niega, por ejemplo, su origen animal o vegetal, e incluso la posibilidad de que se hayan originado a partir de un molde. Tampoco considera que procedan de las manos del Creador desde el principio del mundo, porque en el transcurso de los siglos ya se habrían desgatado y desfigurado, y rechaza asimismo la existencia en este caso de las semillas petrificantes. La razón es que, como se ha observado y comprobado a través de la experiencia, la naturaleza produce cristalizaciones y concreciones en metales, minerales y licores con figuras constantemente uniformes. La hipótesis feijoniana, que considera explicación universal del mecanismo, parte de la idea de que las partículas en estado de fluidez se coagulan mediante la pérdida de movimiento por el enlace entre

(avril, 1729), págs. 654-678; Joseph P. de Tournefort, «Description du labyrinthe de Candie, avec quelques observations sur l'accroissement \& sur la génération des pierres", Mémoires de l'Académie Royale des Sciences de Paris, [s. n.] (1702), págs. 217-234 (concretamente págs. 222-223).

Véanse, además, Gaston Godard, «Le Discours sur les coquilles de mer qu'on trouve en terre ferme, particulièrement en Champagne, adressé à Peiresc dans les années 1630», Travaux du Comité français d'Histoire de la Géologie, 43. ${ }^{\text {a }}$ serie, vol. 18 (2004), págs. 65-78; Marcus Hellyer, «The pocket museum: Edward Lhwys's Lithophylacium», Archives of Natural History, vol. 23, 1 (1996), págs. 43-60; Melvin E. JAHN, «A note on the editions of Lithophylacii Brinnici Ichnographia», Journal of the Society for the Bibliography of Natural History, vol. 6, 2 (1972), págs. 86-97; Peter N. MilLer, Peiresc's Europe: Learning and virtue in the Seventeenth Century, New Haven (CT), Yale University Press, 2000.

29 Manuel Gutiérrez Claverol y Carlos Luque Cabal, Recursos del subsuelo de Asturias, Oviedo, Servicio de Publicaciones de la Universidad de Oviedo, 1993, pág. 289; Manuel Gutí́rrez Claverol, Las aguas termales de Las Caldas de Oviedo, s. 1., Hife, 2014, pág. 60. 
ellas, y de cada combinación, en forma y unión, se derivaría la estructura resultante (TC, VII, 2, § XVI, 75-82).

Sobre la naturaleza orgánica de las conchas marinas y restos de peces petrificados, Feijoo «no permite la menor duda, de que siendo un tiempo individuos de aquellas especies, al tiempo que por quedar en seco les fue faltando la vida y el movimiento, se fueron introduciendo por sus poros varios corpúsculos térreos, o salinos, o metálicos, con que haciéndose como piedras organizadas, se preservaron de corrupción» (TC, VII, 2, § XII, 32). Estas ideas del erudito benedictino están relacionadas a su vez con el origen de las montañas y las acciones del Diluvio bíblico (TC, V, 15, § XV, 18) que se verán en el apartado siguiente.

Finalmente, y en cuanto a la paleontología de vertebrados, Feijoo también participó en el debate sobre la existencia de gigantes humanos en el pasado (TC, $\mathrm{V}$, discursos 1, 15 y 16). La presencia de personajes de gran envergadura es una creencia secular que se remonta hasta las propias raíces de la nuestra civilización, abundan prácticamente en todas las mitologías, y los libros sagrados de todas las culturas están llenos de referencias a estos $\operatorname{seres}^{30}$.

El auténtico debate a este respecto se inició en Europa durante el siglo XVI principalmente con las crónicas procedentes de las Indias Occidentales ${ }^{31}$. Según estos cronistas, el encuentro de los españoles con los patagones de gran estatura, los relatos mitológicos de los indios, y el hallazgo de huesos de gran tamaño, dieron lugar a una importante discusión sobre la existencia de los gi-

30 Véanse unos excelentes estudios histórico-antropológicos en Henri BrESC, «Le temps des géants», Actes des Congrès de la Société des Historiens Médiévistes de l'Enseignement Supérieur Public (13 Congrès, Aixen-Provence, Temps, mémoire, tradition au Moyen-Âge), Aix-en-Provence, Université de Provence, 1982, págs. 243-266; Jean CEARD, «La querelle des géants et la jeunesse du monde», Journal of Medieval and Renaissance Studies, vol. 8 (1978), págs. 37-76.

31 Para un estudio detallado sobre este tema, remitimos a los trabajos de Francisco Pelayo, «El Aparato para la Historia Natural Española de José Torrubia (1698-1761): diluvismo, gigantes y la naturaleza de los fósiles en el pensamiento español del siglo xvıII», en José Torrubia, Aparato para la Historia Natural Española, Madrid, Instituto de Geología Económica / CSIC, 1994 (facsímil de Madrid, Imprenta de los hermanos de Gordejuela y Sierra, 1754), págs. 3-45 (concretamente págs. 14-20); Francisco PELAYo, «El mito de los gigantes americanos. Un debate de la Paleontología de vertebrados española durante la época colonial», en Marie Cécile Bénassy, J. P. Clément, Francisco Pelayo, y Miguel Ángel Puig-Samper (coords.), Nouveau Monde et renouveau de l'Histoire Naturelle, París, Presses de la Sorbonne Nouvelle, 1994, vol. III, págs. 161181; Francisco Pelayo, Del diluvio al megaterio. Los orígenes de la Paleontología en España, Madrid, CSIC, 1996, págs. 28-35; Francisco PeLAyo, «El orden natural y los gigantes: La Gigantologia Spagnola Vendicata (1760) de José Torrubia», Actas XV Jordanas de Paleontología, Simposio del Proyecto PICG 421, Madrid, ITGE, 1999, vol. 26, págs. 685-716 (reproducido en: Archivo Teológico Granadino, vol. 65 (2002), págs. 129-186); Francisco Pelayo, «En busca del hombre antediluviano: los inicios del debate sobre la antigüedad del hombre y la existencia de restos fósiles humanos», Memorias de la Real Sociedad Española de Historia Natural, vol. III (2004), págs. 117-169; Leandro SequeIros y Francisco PeLayo, «Introducción y notas», en José Torrubia, Aparato para la Historia Natural Española, Granada, Universidad de Granada / CSIC, 2007 (facsímil de Madrid, Imprenta de los hermanos de Gordejuela y Sierra, 1754), págs. IX-LXXXI (concretamente págs. XXVII-XXXI). 
gantes en épocas pasadas. Sin embargo, Feijoo, siguiendo las ideas de Tournefort $^{32}$, no da crédito a estas informaciones, aunque muchas de ellas proceden de autores clásicos relevantes como Plinio, Homero o Virgilio, entre otros. Para el benedictino gallego, por ejemplo, los gigantes del estrecho de Magallanes estaban dentro de las noticias fabulosas que llegaban de América, relegando a una cuestión de fe la verdad al respecto, puesto que este tipo de creencias se basaba «únicamente en la autoridad de los hombres; y esta autoridad mal entendida o mal regulada, es quien ha llenado el mundo de fábulas», extravagancias que han pervivido «a la sombra del vano pero ostentoso título de la tradición» (TC, V, 1, § II, 5; TC, V, 16, § I, 2).

A pesar de las referencias que se encuentran en las Sagradas Escrituras sobre la existencia de los gigantes ${ }^{33}$, y el comentario que aporta Agustín de Hipona (354-430 d. C.) en De civitate Dei (c. 419 d. C.) en relación con un molar gigante que se había encontrado en Útica (Túnez) ${ }^{34}$, para Feijoo, desde un punto de vista racional, los huesos hallados de gran tamaño pertenecerían en realidad a otros brutos de mayores dimensiones, que bien podría tratarse de ballenas (TC, I, 12, $\S$ VIII, 30). Todo lo demás, especialmente aquello que gira en torno a la especie humana, sería fantasías imaginativas y extravagantes fabulaciones.

\section{Sobre el origen de las montañas}

La discusión sobre las irregularidades del paisaje se planteaba en términos generalmente teológicos: o bien Dios había creado los montes desde el principio del mundo, o bien creó una tierra plana y las montañas eran fruto de la acción de las aguas diluviales. Estas ideas eran objetables, sobre todo la segunda, porque para Feijoo, basándose una vez más en las Sagradas Escrituras, antes del Diluvio ya había montes, puesto que las aguas cubrieron la tierra unos quince codos por encima de las montañas (Génesis, 7: 19 21).

Feijoo afirmaba que las montañas se habían ido formando por sí mismas de forma gradual a lo largo de muchos siglos (unos 3000 años aproximadamente), y para ello sostenía algunos argumentos: las montañas, por un lado, tenían un

32 Tournefort, «Description du labyrinthe», págs. 222-223.

33 Véanse Génesis, 6: 4; Números, 13: 33; Deuteronomio, 2: 11 y 20; 3: 11 y 13; 1 Samuel, 17: 4-23; 21 : 9. La referencia sobre gigantes que se da a veces para Génesis, 10: 9, es una reinterpretación de un término que se traduce, por ejemplo, como robusto (Vulgata), poderoso (Biblia del Oso), o barragán (Biblia de Ferrara).

34 Agustín de Hipona, La Ciudad de Dios (Libros VIII-XV), Madrid, Gredos, 2012, libro XV, cap. 9, pág. 490; véase además Gaston GoDARD, «The fossil proboscideans of Utica (Tunisia), a key of 'giant' controversy, from Saint Augustine (424) to Peiresc (1632)», en Martina Kölbl-Ebert (ed.), Geology and religion: A history of harmony and hostility, Geological Society of London, Special Publications, 2009, vol. 310, págs. 67-76. 
núcleo interno de piedra, mientras que, por otro lado, estas piedras se generaban mediante determinados tipos de semillas, que crecían y aumentaban. Este aumento paulatino en cuanto a su volumen y tamaño se debía a la existencia de un jugo nutricio o espíritu lapidífico, muy heterogéneo, propio para cada tipo de piedra, que era absorbido, y, en función de la cantidad de esa sustancia y de su capacidad de acción, las montañas adquirían diferentes estructuras y altitudes (CE, III, 30, 54-57).

Ya hemos visto que estas ideas sobre las semillas y el crecimiento las toma Feijoo de diversos autores citados anteriormente como Homberg y Tournefort, pero también y sobre todo de Georgius Baglivi (1668-1707), Giacinto Gimma (1668-1735), y de Nicolas-Claude Fabri de Peiresc (1580-1637) ${ }^{35}$.

Esta posición de Feijoo en relación con las montañas, el diluvio y el hallazgo de los fósiles marinos lo sitúa en una posición concreta en relación con la controversia sobre inmutabilidad de la Tierra: el monje benedictino llega a aceptar que el globo terráqueo ha sufrido grandes cambios o revoluciones que han modificado su fisiografía a lo largo de los siglos, con grandes extensiones de tierra firme que en algún período fueron inundadas por las aguas y que posteriormente se retiraron. Pero niega que el diluvio haya sido el responsable del acarreo de los fósiles hasta las más altas montañas, tal y como defendían muchos autores, entre otros John Woodward (1665-1728), para quien los fósiles eran vestigios de la catástrofe bíblica ${ }^{36}$. Feijoo también se opone, por razones teológicas, a la idea de un planeta en decadencia, donde las montañas no serían otra cosa que las huellas de su propia ruina (TC, III, 15, § XII-XVIII) ${ }^{37}$.

35 Georgii Baglivi, «De vegetationem lapidum», en Opera omnia medico-practica et anatomica, Lugduni [Lyon], J. Posuel, 1703, págs. 475-501; Giacinto GIммa, Della storia naturale delle gemme, delle pietre, e di tutti i minerali, ovvero della fisica sotteranea, Nápoles, G. Muzio, 1730, t. I, libro I, cap. VIII; HomberG, Réflexions; Tournefort, Description, págs. 222-223.

Las ideas de Peiresc están recogidas en Pierre GASsend, Peiresc 1580-1637: vie de l'illustre NicolasClaude Fabri de Peiresc conseiller au parlement d'Aix, París, Belin, 1999, y, especialmente, aunque doscientos cincuenta años más tarde, en Phillipe Maizey de Larroque, Notes inédites de Peiresc sur quelques points de l'Histoire Naturelle, Digne, Chaspoul et Barbaroux, 1896, caps. III y VII. Véanse, además, Gaston GoDARD, «Peiresc, Gassendi, Menestrier, La Ferrière, Gilles de Loches...: Un cercle méconnu de "géologues" au début du dix-septième siècle», Travaux du Comité Français d'Histoire de la Géologie, 3. " serie, vol. 10 (1996), págs. 155-163; Miller, Peiresc's Europe; Pelayo, «El Aparato», págs. 20-33; Pelayo, Del Diluvio, págs. 112-138; SEQueIros y Pelayo, «Introducción y notas», págs. Xxxv-XuIV.

36 John Woodward, An Essay towards a Natural History of the Earth, Londres, R. Wilkin, 1695 (existe trad. francesa: Géographie Physique, ou Essai sur l'Histoire Naturelle de la Terre, Amsterdam, aux dépens de la Compagnie, 1735); Feijoo parece no haber consultado esta obra, de la que tan solo hace referencia a la extensa noticia que se da en las Mémoires de Trévoux (février, 1736, artículo XVII, págs. 244-261) de la versión francesa (TC, IV, 5, § VI, 28).

37 Sobre la decadencia de la Tierra y el origen de las montañas, véanse Capel, La Física, cap. 5, págs. 82-105; Gordon L. DAvies, The Earth in decay: A history of British geomorphology, 1578 to 1878, Londres, Mcdonald, 1969; Cándido M. García Cruz, «El origen de las montañas. I. Del mito y la superstición 


\section{Sobre terremotos y volcanes}

Tras el gran terremoto que asoló Lisboa el 1 de noviembre de 1755, y que además afectó o se sintió en buena parte de España y del resto de Europa, así como en el norte de África, numerosos autores se implicaron en el análisis y discusión de las causas de los temblores de tierra con la publicación de importantes trabajos sobre el tema que se consideran un preámbulo del nacimiento de la sismología como ciencia ${ }^{38}$.

Feijoo se hizo eco de esta catástrofe a través de la correspondencia que mantuvo con don Joseph Díaz de Guitián, vecino de la ciudad de Cádiz (fechada entre el 19 de noviembre de 1755, y el 13 de enero de 1756), y otra carta dirigida a Joseph Rodríguez de Arellano, canónigo de la catedral de Toledo (del 25 de enero de 1756), que se publicaron originalmente en 1756 en un pequeño estudio bajo el título de Nuevo systhema sobre la causa physica de los terremotos, y que luego aparecerían, en 1760, en el tomo V de sus Cartas eruditas y curiosas (CE, V, 25-29). En esta edición, se incluye una breve carta que sirve de introducción a sus ideas concernientes a los terremotos; en ella hace algunos comentarios sobre ciertas señales previas o pronósticos que bien podrían anteceder a los temblores de tierra, porque «nos importa infinitamente más conocer las señales, que preceden a los Terremotos (si hay algunas seguras), que indagar

al neptunismo", Enseñanza de las Ciencias de la Tierra, vol. 15, 1 (2007), págs. 16-29 (preferentemente págs. 20-26).

38 Entre estos trabajos destacan Élie BERTRAnd, Mémoires historiques et physiques sur les tremblemens de terre, La Haya, Pierre Gosse, 1757, mémoires I, VI y VII; John Bevis (ed.), The history and philosophy of earthquakes, from the remotest to the present time, Londres, J. Nourse, 1757 (ed. moderna en Cambridge, Cambridge University Press, 2013); véase, además, Charles Davison, The founders of seismology, Nueva York, Cambridge University Press, 2014; John MichelL, «Conjectures concerning the cause, and observations upon the phaenomena of earthquakes; particularly of that great earthquake of the First of November, 1755, which proved so fatal to the city of Lisbon, and whose effects were felt as far as Africa, and more or less throughout almost all Europe», Philosophical Transactions of the Royal Society of London, vol. 51 (1760), págs. 566-634; véase, también, Mark MoLesky, This gulf of fire. The great Lisbon earthquake, or Apocalypse in the age of science and reason, Nueva York, Penguin, 2015. Sobre las distintas explicaciones históricas del origen de los terremotos y volcanes, véanse Emanuela Guidoboni, «Earthquakes, theories from 1600 to 1800» y «Earthquakes, theories from antiquity to 1600», en Gregory A. Good (ed.), Sciences of the Earth: An encyclopedia of events, people, and phenomena, Nueva York, Garland, 1998, vol. I, págs. 197-214; Erhard OEsER, «Historical earthquake theories from Aristotle to Kant», en R. Gutdeutsch, Gottfried Grünthal y Roger M. W. Musson (eds.), Historical earthquakes in Central Europe, Viena, Abhandlungen der Geologischen Bundesanstalt, 1992, vol. I, págs. 11-31; David R. OLdroyd, Thinking about the Earth: A history of ideas in Geology, Londres, Athlone, 1996, cap. 2; Grégory QuenET, Les tremblements de terre aux XII et XVIII siècles. La naissance d'un risque, Seyssel, Champ Vallon, 2005; y, en relación expresamente con España, Horacio CAPEL, «Organicismo, fuego interior y terremotos en la España del siglo XVIII», GeoCrítica, 27 / 28 (1980), [s. n.].; Agustín UdíAs, «Earthquakes as God's punishment in 17th- and 18th-century Spain», en Martina Kölbl-Ebert (ed.), Geology and religion: A history of harmony and hostility, London, Geological Society of London, 2009, vol. 310, págs. 41-48. 
sus causas» $(\mathrm{CE}, \mathrm{V}, 13,3)^{39}$. Entre estas señales destaca la turbación o secado del agua de fuentes y pozos, el olor y el sabor del agua, la intumescencia y agitación del mar, la fuga de aves y otros animales terrestres, algunos fenómenos atmosféricos como columnas ígneas, una línea delgada blanca en el horizonte, la claridad y serenidad en el aire, o ruidos subterráneos. De todos estos, que la ciencia actual denomina precursores sísmicos, Feijoo duda de la mayoría, le resultan inciertos, a pesar de que el primero de ellos, relativo al agua de las fuentes, tenga una tradición muy antigua, tal y como lo reconoce el propio autor citando a varios escritores latinos, como Marco Tulio Cicerón (106-43 a. C.) en De divinatione, o Plinio el Viejo (23-79 d. C.), en Naturalis Historia ${ }^{40}$.

Tan solo el olor y sabor del agua le parecen viables, porque las considera fruto de las fermentaciones minerales que se producen en el interior del planeta. Estas ideas constituyen una parte de la base de las explicaciones que realiza Feijoo sobre las causas de los terremotos puesto que se derivan de la teoría físico -química sobre el origen de los temblores de tierra que predominaba en esa época, en competencia con la idea de la virtud eléctrica ${ }^{41}$, y que se debe a Nicolas Lémery (1645-1715), con la que intentaba explicar además los fuegos subterráneos, los rayos, los relámpagos y los truenos ${ }^{42}$. Esta teoría partía de la creencia de que el planeta estaba horadado por multitud de cavernas, canales y hendiduras por las que podían circular el fuego, el agua y el aire a altas temperaturas, emanaciones que eran las causantes de los terremotos y las erupciones volcánicas. La energía mecánica o térmica necesaria para tales efectos se encontraba en las fermentaciones minerales o materias inflamables que existían en el interior de la tierra, especialmente en ciertos derivados del hierro y del azufre como las piritas. Esta es la idea fundamental a la que alude Feijoo sin citar a Lémery, pero sí utiliza la obra Diálogos físicos (Entretiens physiques ${ }^{43}$ del jesuita Noël Regnault (1683-1762) como fuente de la teoría físico-química

39 En última instancia, tal y como lo expresó Joseph Cevallos, censor del Nuevo Systhema, «Es dogma de Fè Catholica, que Dios produce todas las causas, y efectos: y siendo efectos naturales los Terremotos, truenos, y tempestades, concurre Dios à su produccion, como à otro qualquier efecto natural» (FEJjoo, Nuevo Systhema, pág. [19], [ortografía original]).

40 Marco T. Cicerón, Sobre la adivinación, Madrid, Gredos, 1999, libro I, cap. 50, párrafo 112; Plinio EL VIEjo, Historia Natural, Madrid, Gredos, 1995, libros I-II, 2, cap. 79.

41 CAPEL, «Organicismo, fuego interior y terremotos».

42 Nicolas LÉMERY, «Explication physique et chymique des Feux souterrains, des Tremblemens de Terre, des Ouragans, des Eclairs \& du Tonnerre», Mémoires de l'Académie Royale des Sciences de Paris, 1703, págs. 101-110; véase la traducción castellana de esta obra en Cándido M. GARCía Cruz, «Nicolas Lémery (1645-1715) y su teoría físico-química sobre diversos fenómenos de interés para las Ciencias de la Tierra», Cuadernos Dieciochistas, vol. 16 (2015), págs. 311-337.

43 Noël Regnault, Entretiens physiques d'Ariste et d'Eudoxe, París, J. Clouzier, 1732, t. II, viII Entretien, págs. 180-197. 
lemeriana ${ }^{44}$. Sin embargo, para el benedictino, esta teoría solo sería aplicable a los terremotos que tienen su origen en la incensión de las materias inflamables contenidas en las cavidades subterráneas situadas en la zona más superficial de la corteza terrestre; para los grandes terremotos, como el de Lisboa, habría que buscar las causas más abajo, a mayor profundidad ${ }^{45}$. En este caso, las materias inflamables deben localizarse a una distancia considerable del centro de la Tierra, agitadas por los fuegos subterráneos, de donde surgen abundantes exhalaciones que dan lugar a tempestades impetuosas, mayores incluso que las tormentas que forman en la atmósfera los truenos, los rayos y los relámpagos ${ }^{46}$.

Aun así, Feijoo plantea otra explicación para estos grandes terremotos a través del fenómeno de la electricidad que venía estudiándose desde hacía algunas décadas.

A partir de los experimentos realizados en Norteamérica a finales de la primera mitad del siglo XVIII por Benjamin Franklin (1706-1790) sobre la electricidad en la atmósfera ${ }^{47}$, William Stukely (1687-1765) desarrolló su teoría sobre el origen de los terremotos basándose en dicho fenómeno: según su parecer, el interior de la Tierra debía ser más bien sólido, y no existían cavidades lo suficientemente amplias donde se produjeran grandes explosiones que dieran lugar a la fuerza necesaria para los temblores de tierra ${ }^{48}$. Para Stukely, los terremotos se producían por una descarga eléctrica de la misma naturaleza que la que ocurría en los experimentos sobre la electricidad. La Tierra poseía un estado eléctrico dispuesto para una vibración particular de esta naturaleza, tal y como se podía concluir de una serie de fenómenos frecuentes que anteceden a los temblores de tierra, como la frescura de las plantas, la luminosidad septentrional, y espe-

44 Feijoo, Nuevo Systhema, pág. 1; CE, V, 25, 1.

45 Fejoo, Nuevo Systhema, págs. 5 y 17; CE, V, 25, 1 y 5 . Esta es otra observación interesante de Feijoo y también de una gran modernidad, puesto que la geología actual relaciona la intensidad de los seísmos con la profundidad del hipocentro.

46 FeIjoo, Nuevo Systhema, págs. 15, 17 y 18; CE, V, 29, 2, 6 y 8.

47 Estos experimentos se encuentran descritos en la correspondencia que mantuvo Franklin con Peter Collinson (1694-1768) durante 1747; véase Peter CoLlinson, Experiments and observations on electricity, made at Philadelphia in America, by Mr. Benjamin Franklin, Londres, E. Cove, 1751, págs. 4-9; esta correspondencia está reproducida en Jared Sparks, The works of Benjamin Franklin, Boston (MA), Tappan \& Whittemore, 1837, vol. V, págs. 180-340 (experimentos en págs. 192-196).

48 William StuкеLy, «On the causes of earthquakes», Philosophical Transaction of the Royal Society of London, vol. 46, 491 (1749), págs. 641-646; «Concerning the causes of earthquakes», Philosophical Transaction of the Royal Society of London, vol. 46, 491 (1749), págs. 657-669; «The philosophy of earthquakes», Philosophical Transaction of the Royal Society of London, vol. 46, 491 (1749), págs. 731-750 (reproducido en Bevis The history and philosophy of earthquakes, págs. 253-278); véanse, además, David B. НАYсоск, William Stukely, science, religion and archaeology in Eighteen Century-England, Woodbridge, Boydell Press, 2002, cap. 4; Jean-Paul PorRIER, «Electrical earthquakes: A short-lived theory in the 18th century», Earth Sciences History, vol. 35, 2 (2016), págs. 283-302. 
cialmente la Aurora australis, que se traslada hacia el sur al contrario de lo que es habitual, con unos colores nunca vistos ${ }^{49}$. Para que se produzca un terremoto sería necesario el contacto con algún cuerpo no eléctrico, necesariamente $a b$ extra, procedente de la atmósfera, como por ejemplo las nubes, de acuerdo con los experimentos de Franklin de 1747, bien surgidas del mar o de las exhalaciones de la tierra. Al igual que se producen los truenos en la atmósfera, para Stukely, cuando una nube no eléctrica descarga su contenido sobre cualquier zona de la tierra altamente electrizada, tiene lugar un terremoto.

Aunque no cita los trabajos de Stukely que se han comentado, los argumentos a los que recurre Feijoo poseen los mismos fundamentos. El punto de partida es la imposibilidad de que un temblor de tierra, como el de la capital portuguesa, que fue percibido, como ya se ha comentado, también en casi toda Europa y el norte de África casi simultáneamente, hubiese sido causado y transmitido a distancias considerables de forma instantánea por la combustión de materias inflamables contenidas en las cavernas de la tierra próximas a la superficie ${ }^{50}$. Como fuente documental para su Systhema cita el Ensayo de Jean Antoine Nollet (1700-1770), que también había realizado diversos experimentos sobre la electricidad ${ }^{51}$. De acuerdo con dichos experimentos, las manipulaciones eléctricas producen un fuego que estaría difundido por todos los cuerpos, incluida el agua, pero en mayor proporción en los sulfúreos y bituminosos. Quizás lo más importante de estas experiencias era lo que se conocía como golpe fulminante o experiencia de Leyde (Leiden), realizado por primera vez, en 1746, por Pieter van Musschembroek (1692-1761), y que significó el descubrimiento del condensador eléctrico al que se denominó en un principio Botella de Leiden ${ }^{52}$. Su aspecto más admirable era la capacidad de propagación del fenómeno eléctrico en forma de fulminación a través de numerosas personas enlazadas entre sí a través de sus manos, sin pérdida de efecto ni de intensidad. Teniendo en cuenta, por otro lado, la duración de los volcanes durante siglos, Feijoo supone que la materia eléctrica (constituida fundamentalmente por sustancias sulfúreas y bituminosas) en el interior profundo del planeta debe ser abundante; dicha materia eléctrica puede ser agitada por cualquier causa, lo cual impele una inmensa fuerza que provoca

49 Ninguno de estos fenómenos atmosféricos citados por STUкELY, «On the causes of earthquakes», p. 642, son auténticos precursores sísmicos.

50 FeiJoo, Nuevo Systhema, págs. 22-32; CE, V, 29, 15-35.

51 Jean A. Nollet, Ensayo sobre la electricidad de los cuerpos, Madrid, Imprenta del Mercurio, 1747; Jean A. NoLlet, Recherches sur les causes particulières des phénomènes électriques, París, Guerin, 1749; véase, además, Isaac Benguigui, Théories électriques du XVIII siècle, Ginebra, H. Georg, 1984.

52 Petro van Musschenвroek, Institutiones physicae, Lugduni Batavorum [Leiden], S. Luchtmans, 1748, cap. XVII § 562-635; véase también NoLLET, Ensayo, págs. 76-77. 
vibraciones y que carece de límites, recorriendo enormes distancias sin mermar en modo alguno su ímpetu y sus efectos. Así se justificaría el poder sentir un gran temblor de tierra en zonas muy distintas y distantes, casi al mismo tiempo ${ }^{53}$.

\section{Sobre los puentes intercontinentales}

Las cartografías que se derivaron tras el descubrimiento de América, y las expediciones transoceánicas que se organizaron a su vez como consecuencia de ello, en especial aquellas que tenían intereses científicos, desvelaron la presencia humana, así como la existencia de una flora y una fauna en regiones aisladas físicamente muy distintas de las que se encontraban en las tierras conocidas. Esto dio lugar a uno de los debates más interesantes sobre el poblamiento de estos territorios, preferentemente del Nuevo Mundo. Los primeros planteamientos que se hicieron, y que habrían permitido las migraciones, datan de finales del siglo XVI, cuando el jesuita español José de Acosta (1540-1600), en su Historia natural y moral de las Indias (1590) ${ }^{54}$, «conforme a razón y al orden y estilo de las cosas humanas» y sin necesidad de recurrir a la intervención divina, sugirió que el poblamiento de las Américas había sido a través de conexiones por tierra $^{55}$. Esto dio pie a que se propusieran ideas parecidas en la segunda mitad del siglo XVII, y que explicaban la existencia de los seres humanos, animales y plantas, mediante el paso a través de la congelación de ciertos mares según Georgius Hornius, nombre latinizado de Georg Horn (1620-1670), en su De

53 Estas ideas de Feijoo sobre el origen de los terremotos fueron copiadas por M. Isnard ese mismo año, aunque no lo cita en ningún momento; véase M. Isnard, Mémoires sur les tremblemens de terre, París, Veuve David jeune, 1758 (existe un amplio comentario de esta obra en Journal de Sçavans, vol. 37 (1758), págs. 68-76).

$54 \quad$ No es posible considerar El Paraíso en el Nuevo Mundo (escrita entre 1645 y 1650) de Antonio de León Pinelo (1590-1660) como el primer antecedente de las ideas sobre el poblamiento de América, puesto que este autor lo que plantea en el segundo volumen de la obra citada es que el Jardín del Edén se encontraba en Sudamérica, y era, por lo tanto, el centro de dispersión de la especie humana; véase Antonio de León Pinelo, El Paraíso en el Nuevo Mundo. Comentario apologético, Historia Natural, y Peregrina de las Indias Occidentales, Islas i Tierra-Firme del Mar Océano, Madrid, [s. n.], 1656 (sólo portada e índices: edición completa póstuma en Lima, Comité del iv Centenario del Descubrimiento del río Amazonas, 1943). Véanse, además, Rosa Peldicer Domingo, «Continens Paradisi: El libro segundo de El Paraíso en el Nuevo Mundo de Antonio de León Pinelo», América sin Nombre, 13-14 (2009), págs. 30-36; Carlos Rey Pereira, «El Paraíso en el Nuevo Mundo. Entre el ejemplo y la excepción», Cuadernos para la Investigación de la Literatura Hispánica, 29 (2004), págs. 141-160.

55 José de Acosta, Historia natural y moral de las Indias, Las Rozas, Dastin, 2002, libro I, cap. XVI (pág. 97) y cap. xx. 
originibus americanis ${ }^{56}$, o de auténticas conexiones terrestres, bien físicamente a lo largo de sus litorales, o a través de puentes intercontinentales, como postulaba Matthew Hale (1609-1676) en The primitive origination of mankind ${ }^{57}$. La gran mayoría de los puentes habrían sido estructuras episódicas, y por lo tanto no permanentes, puesto que las evidencias indicaban que ya no existían como tales, exceptuando casos muy concretos en forma de istmos geográficos, como el de Panamá o Bering, que con el tiempo se denominarían eslabones ístmicos. Consecuentemente, debían de haberse hundido en las cuencas oceánicas grandes volúmenes de materiales continentales mediante cataclismos; este fenómeno, además, tuvo que haber sido cíclico, es decir, la gran mayoría de las conexiones terrestres se habrían formado y destruido repetidamente, explicándose así las similitudes biológicas de diferentes épocas en la historia de la Tierra, y permitiendo al mismo tiempo el aislamiento geográfico durante largos períodos de tiempo ${ }^{58}$.

Feijoo recoge esta misma idea, aunque la expresa, en principio, de una forma un poco ambigua: por un lado, duda de estas conexiones entre Europa y América porque las «innumerables relaciones de viajes marítimos destruyen la sospecha de comunicación por tierra entre los dos Continentes» (TC, V, 15, § V, 8-9), mientras sostiene, por otro lado, que «la disposición exterior del Orbe Terráqueo es hoy bastante distinta de la que hubo en otro tiempo. Puesto esto, es fácil concebir, que aunque hoy los dos Continentes están separados, en los tiempos antiquísimos estuvieron unidos, o se comunicaban por tierra» (TC, V, 15, § VIII, 16). Feijoo prefiere esta causación natural antes que sucesivas creaciones por parte de Dios, incluyendo aquí la teoría de los preadamitas de Isaac La Peyrère (1596-1670) (TC, V, 15, § II-VI) ${ }^{59}$, y alguna otra opción providencial a la que solo se debería recurrir en caso de extrema necesidad, como sería el caso del ministerio de los Ángeles (TC, V, 15, § VI, 13) ${ }^{60}$.

56 Georgius Hornius, De originibus americanis, Hagae Comitis [La Haya], Adriani Vlaco, 1652, libro III, cap. VI.

57 Matthew Hale, The primitive origination of mankind, Londres, W. Shrowsbery, 1677, sec. II, caps. VII y IX.

58 Cándido M. García Cruz, «Puentes intercontinentales e isostasia: Aspectos históricos y didácticos», Enseñanza de las Ciencias de la Tierra, vol. 6, 3 (1998), págs. 211-216.

59 Sobre los preadamitas, véase Isaac La Peyrère, Praeadamitae, Amsterodami [Amsterdam], L. \& D. Elzevier, 1655 (existen traducciones inglesa, Man before Adam, Londres-Hildesheim, G. Olms, 1656, e italiana moderna: I preadamiti. Macerata, Quodlibet, 2004); véase, además, David N. Livingstone, Adam's ancestors: race, religion, and the politics of human origins, Baltimore, Johns Hopkins University Press, 2008.

${ }^{60}$ Ésta es una clara referencia bíblica al traslado del profeta Habacuc por parte de un ángel desde Judea hasta Babilonia para llevar alimentos al también profeta Daniel, y al que devolvió nuevamente a Judea (Daniel, 14: 33-39). 


\section{A modo de conclusión}

La discusión crítica de la realidad cultural española, sensu lato, heredada de los Novatores y su difusión, fue continuada desde su cátedra de Teología de la Universidad de Oviedo por Benito Jerónimo Feijoo a lo largo de la primera mitad del siglo XVIII. Dentro de las ciencias naturales, la geología ocupó una parte importante tanto en los ensayos de su Teatro crítico universal como en la correspondencia que forma sus Cartas eruditas y curiosas. Analizó diversos temas que se planteaban en esa época como controversias de gran interés y que fueron conformando la geología como ciencia independiente, desde el origen de la Tierra y la formación de las montañas, hasta el significado y origen de los fósiles y el Diluvio universal, las causas de los terremotos y de los volcanes, y las conexiones intercontinentales. Aun cuando estuvo sujeto al dogma escritural, Feijoo siempre mostró su adhesión inquebrantable a la razón y la experiencia como fuentes de certeza para el conocimiento por encima de los postulados aristotélicos y las creencias comunes basadas en la tradición. Dentro de la corriente mecanicista, refutó la teoría de los torbellinos cartesiana para explicar el origen de la Tierra, aceptó la idea del fluido lapidífico para la génesis de las montañas y como origen de los fósiles, que además consideraba de naturaleza orgánica, y la explicación físico-química para los terremotos más pequeños y los fuegos subterráneos, mientras que los grandes temblores de tierra, y sobre todo su transmisión a grandes distancias, los asociaba con fenómenos eléctricos que se derivaban de las profundidades del planeta. Se mantuvo siempre al corriente de las ideas científicas de su época a través de algunas revistas y misceláneas importantes francesas, pero también utilizó como fuentes de documentación algunas obras de autores relevantes, tanto en francés como otras escritas en latín. 